\title{
Gewerkschaften in Indonesien
}

\author{
von Eva-Maria Garang-Schaarschmidt
}

\section{Kurze Einführung in die Entwicklungsgeschichte der indonesischen Gewerkschaftsbe- wegung}

Die Entstehung der indonesischen Gewerkschaftsbewegung steht in engem Zusammenhang mit dem Kampf des unter der holländischen Kolonialherrschaft, die sich seit Beginn des 17. Jahrhunderts schrittweise etabliert hatte, leidenden Volkes um nationale Unabhängigkeit. War der Widerstand bis zu Beginn des 20. Jahrhunderts militärischer Art gewesen, in Form lokaler und regionaler Aufstände, die immer wieder niedergekämpft worden waren, so begannen nun die in wachsendem Maße zur Verwaltung des Landes mit herangezogenen indonesischen Angestellten, sich politischer Mittel zu bedienen. Da Vereinigungen und Versammlungen politischer Art aber bis 1915 strikt verboten waren (das entsprechende Gesetz wurde erst 1919 aufgehoben), ${ }^{1}$ gründeten sie zunächst kulturelle Vereinigungen (Budi Utomo, 1908) und traten holländischen Gewerkschaften (ab 1905) bei, soweit diese überhaupt indonesische Angestellte aufnahmen. Die ersten revolutionären gewerkschaftlichen Aktionen entwickelten sich in Zusammenarbeit mit holländischen Kommunisten und Sozialisten und kulminierten in der 1926/27er Rebellion der indonesischen Kommunisten, die ebenfalls von der holländischen Kolonialregierung niedergeschlagen wurde.

Die islamisch ${ }^{2}$ orientierten Führer in der nationalistischen Bewegung, die teils mit den kommunistischen zusammengearbeitet, zum größeren Teil aber in scharfer Konkurrenz zu ihnen gestanden hatten, verlegten sich nun mehr auf nicht-politische Aktivitäten. ${ }^{3}$ Dies taten auch die vielen rein indonesischen Gewerkschaften, die sich inzwischen gebildet hatten und in der Folge bildeten, bis im Februar 1942 der Einmarsch der Japaner die gesamte politische Situation änderte. Die Holländer hatten sich ohne nennenswerten Widerstand ergeben. Die Japaner verboten alle gewerkschaftlichen und sonstigen indonesichen Organisationen ${ }^{4}$ und zwangen Indonesien in ihre Kriegsmaschinerie. Aber es gelang den indonesischen Nationalisten, diese Situation zu nutzen und im August 1945 sofort nach der Kapitulation der Japaner als Ausgangspunkt für die nationale Unabhängigkeit zu machen. Sofort nach der Unabhängigkeitserklärung entstanden überall in In-

1 Tedjasukmana, Iskandar: The Political Character of the Indonesian Trade Union Movement, Ithaca, N.Y., 1958, S. 3.

2 Nominell gehören fast $90 \%$ der indonesischen Bevölkerung dem Islam an. Nur ein kleiner Teil der Muslime vor allem nicht aus Java stammende - können aber als strenggläubig betrachtet werden.

3 Kahin, George McTuhan: Nationalism and Revolution in Indonesia, Cornell University Press, Ithaca, N.Y., 1952, S. 87.

4 Tedjasukmana, op. cit., S. 17. 
donesien spontan Arbeiterorganisationen, um die Betriebe und Gesellschaften von den Japanern zu übernehmen. War in der Periode vor dem II. Weltkrieg ein Antagonismus zwischen Islam und Kommunismus gegeben, so entstand nun, als, noch während die Indonesier ihre Unabhängigkeit gegen die mit ihren alten Machtansprüchen zurückkehrenden Holländer verteidigen mußten, in der sogenannten Madiun-Affäre sich die Kommunisten gegen die junge Republik wandten, der Antagonismus zwischen Kommunisten und dem Militär. Die vier wesentlichen, den weiteren Werdegang Indonesiens bis 1965 beherrschenden Kräfte hatten sich also bereits 1950 etabliert: die kommunistische Partei, die nationalistische Partei, die islamischen Parteien und das Militär, jeweils mit ihnen angeschlossenen oder unter ihrem Einfluß stehenden Gewerkschaftsbünden. Dem charismatischen nationalistischen Präsidenten Sukarno gelang es mit seinen synkretistischen Ideologien, wie Pancasila, Marhaenismus, nicht, einen Ausgleich zwischen diesen Kräften herzustellen. Auch sein Versuch, 1959 den drängenden Problemen durch eine "Gelenkte Demokratie "s zu begegnen, in der er noch mehr als zuvor zu diktatorischen Maßnahmen griff, verstärkte nur die Gegnerschaft zwischen den beiden Blöcken: Gegen die Zusammenarbeit von Sukarno und seiner nationalistischen Partei mit der Kommunistischen Partei Indonesiens auf der einen Seite rückten auf der anderen Seite das Militär und die islamischen Parteien, mit ihnen die islamischen Gewerkschaften, näher zusammen.

Die wirtschaftliche Lage hatte sich immer weiter verschlechtert, da die Kräfte des indonesischen Volkes einmal durch die Aufrechterhaltung der Einheit des indonesischen Staates gegen separatistische Bewegungen, zum anderen aber auch durch außenpolitische Kampagnien (Malaysia, West-Irian), mit denen Sukarno den revolutionären Elan aus der Zeit des Unabhängigkeitskampfes lebendig erhalten wollte, erschöpft waren. Als Sukarno 1965 erkrankte und sich sein Zustand verschlechterte, traten die Spannungen innerhalb der Gesellschaft offener zutage. Innerhalb des Militärs wollte eine Gruppe kommunistisch orientierter Offiziere unter Col. Untung mit einem Coup d'Etat ${ }^{6}$ einem vermuteten Staatsstreich einiger Generäle zuvorkommen. Dieser Coup wurde vom übrigen Militär unter Führung von Suharto niedergeschlagen, und dies war der Anlaß für die schon lange zu den nationalistischen und v. a. kommunistischen Gruppen im Lande in Opposition stehenden Militärs und islamischen Gruppen, sich ihrer ideologischen Gegner zu entledigen, ${ }^{7}$ so auch der kommunistisch orientierten Gewerkschaften.

Mit dieser knappen Andeutung der historischen Entwicklung, die in dem hier gesteckten Rahmen natürlich nicht einmal annähernd die komplexen Abläufe der neueren Geschichte Indonesiens und seiner Gewerkschaften wiedergeben kann, sollte nur auf drei der wichtigsten Faktoren der indonesischen Gewerkschaftsbewegung aufmerksam gemacht werden:

5 In diesem Zusammenhang entwarf Sukarno das Konzept einer Einteilung der Gesellschaft in "Funktionale Gruppen", auf dem auch die heutige Regierungspartei GOLKAR aufbaut.

6 Auch nach dem Tag, an dem er stattfand, 30. 9., "Gerakan Tiga Puluh September" = GESTAPU genannt.

7 Prah, Kwesi: The Social Background of Coup d'Etat (Brazil, Indonesia and Ghana), Amsterdam, 1973, S. $50 \mathrm{f}$. 
1. Die Gewerkschaften wurden sehr oft ${ }^{8}$ nicht als Selbsthilfegruppen der Arbeiter und Angestellten von der Basis her aufgebaut, sondern von politisch engagierten Intellektuellen, meist führende Mitglieder einer Partei, von oben.

2. Damit hängt zusammen, daß sich die Gewerkschaften, wann immer sie sich einigermaßen frei entfalten konnten, nicht nur als Kampforganisationen für bessere Arbeitsbedingungen verstanden haben, sondern auch als politische Organisationen.

3. Die ideologischen und politischen (ja, sogar die gesetzlichen) Grundlagen, Pancasila und die Gelenkte Demokratie, in deren Rahmen auch der neue indonesische Gewerkschaftsbund FBSI entstanden ist, sind bereits lange vor der sogenannten "Neuen Ordnung" (orde baru) gelegt worden.

\section{Die wichtigsten Gewerkschaftsbünde zwischen 1945 und 1965}

Im folgenden seien kurz die wichtigsten Gewerkschaftsbünde, wie sie bei Ausbruch des Coup d'Etat 1965 bestanden, beschrieben.

\subsection{Die kommunistischen Gewerkschaftsbünde SOBSI}

Der kommunistische Gewerkschaftsbund SOBSI (Sentral Organisasi Buruh Seluruh Indonesia, Zentrale der Gewerkschaften Ganz Indonesiens) ist der mitgliederstärkste, ${ }^{10}$ best organisierte und wirksamste Gewerkschaftsbund dieser Periode gewesen. ${ }^{11}$ Er ging 1946 aus der Mehrzahl der nach 1945 überall in Indonesien spontan entstandenen Gewerkschaften hervor. 1948 nahmen viele seiner Mitglieder an der Madiun-Affäre teil. Da aber kein anderer Gewerkschaftsbund über eine Massenbasis, wie sie SOBSI innerhalb kürzester Zeit geschaffen hatte, verfügte, konnte es sich von dieser Niederlage rasch erholen: 1956 waren bereits ca. $60 \%$ aller organisierten Arbeiter Mitglied bei SOBSI oder einer der ca. 40 an SOBSI angeschlossenen Einzelgewerkschaften, die es in allen Branchen gab. Obwohl SOBSI eine von der Kommunistischen Partei Indonesiens (PKI) unabhängie Organisation war, waren Organisationsstruktur (Demokratischer Zentralismus) und Ziele (Errichtung einer sozialistischen Gesellschaft mit dem Mittel des Klassenkampfes, Bekämpfung von Imperialismus und Kapitalismus) doch denen der PKI

8 V. a. die nichtkommunistischen.

9 Z. B. durch die 1959 erfolgte Wiedereinsetzung der Verfassung von 1945, die dem Präsidenten weite Machtbefugnisse einräumt; auch der überwiegende Teil der Arbeitsgesetzgebung stammt aus der Zeit der norder lama", der alten Ordnung. Vgl. Sudono, Agus: Sejarah, Kelahiran dan Perkembangan FBSI, Jakarta 1978 (Entstehungsgeschichte und Entwicklung von FBSI) S. 15.

10 Mitgliederzahlen anzugeben, ist nicht sehr sinnvoll, da sie jeglicher nachprüfbaren Grundlage entbehren. Die Gewerkschaften machten ihre Angaben nach eigenen Schätzungen, die bis auf wenige Ausnahmen maßlos übertrieben waren. Man kann sich nur an Relationen halten.

11 Von Hawkins wurde SOBSI sogar als der einzige ef fektive Gewerkschaftsbund Indonesiens bezeichnet. Hawkins, Everett D.: Labor in Transition, in: McVey, Ruth (ed.): Indonesia. New Haven, 1967, S. 248-271, S. 259. 
analog, viele seiner Führer und Mitglieder waren gleichzeitig Führer oder einfache Mitglieder von PKI. ${ }^{12}$ So wurden Strategie und Politik von SOBSI im allgemeinen von der PKI definiert. ${ }^{13}$

Da im Zuge der Úbernahme holländischer Firmen viele Militärs in leitenden Positionen gelangten, wurden sie zu Gegnern der betreffenden Arbeiter, die für diese Úbernahmen gekämpft hatten. Besonders aber durch die Pläne und Bemühungen, durch Bewaffnung der Arbeiter und Bauern eine Volksmiliz zu schaffen, setzte sich PKI, mit ihr der angeschlossenen Bauernverband und SOBSI, in erbitterte Gegnerschaft zum Militär.

Und so wurden auch die Führer und Mitglieder von SOBSI im Zuge der Kommunistenverfolgung nach dem Coup d'Etat bekämpft, getötet und inhaftiert, zumindest aber mit Organisationsverbot belegt, obwohl es keinerlei Hinweise auf eine Beteiligung von SOBSI am Coup d'Etat gibt.

Bei aller berechtigten Kritik an SOBSI, das of t zu recht militanten Mitteln griff, um seine bedeutende Position in der Arbeiterbewegung auszubauen, muß doch betont werden, daß mit ihr der größte und eigentlich einzige Gewerkschaftsbund, dessen ihm angeschlossene Gewerkschaften echte und aktive Arbeiterorganisationen waren, ausgelöscht, die Arbeiterbewegung ihrer fähigsten Kader beraubt und ein Wiederaufleben einer von der Basis ausgehenden Arbeiterbewegung auf lange Zeit hin unmöglich gemacht wurde.

\section{SOBRI}

Der ebenfalls kommunistische, aber sehr kleine, seit 1952 bestehende Gewerkschaftsbund SOBRI (Sentral Organisasi Buruh Republik Indonesia, Zentrale der Gewerkschaften der Republik Indonesien), der trotzkistischen Partei Murba zugehörig, existierte weiter, er hatte auch nie eine große Bedeutung gehabt und ordnete sich nach 1965 den Zentralisierungsmaßnahmen der Regierung unter.

\subsection{Die nationalistischen Gewerkschaftsbünde}

Die bedeutende Rolle, die die nationalistische Partei, die Partei Sukarnos, PNI, in der politischen Landschaft Indonesiens gespielt hat, konnten ihre Gewerkschaftsorganisationen nie erreichen. Erst Ende 1952, als SOBSI schon zur beherrschenden Kraft in der Gewerkschaftsbewegung herangewachsen war, schuf sich PNI mit KBKI (Kesatuan Buruh Kerakyatan Indonesia, Vereinigung Demokratischer Arbeiter Indonesiens) eine Massenbasis, v. a. um bei Wahlen die Unterstützung städtischer und ländlicher Arbeiter und Angestellter zu gewinnen. ${ }^{14}$ Besonders bei diesem Gewerkschaftsbund wurde die Natur vieler indonesischer Gewerkschaften, als Gebilde politischer Parteien, geschaffen

12 U. a. Tedjasukmana, op. cit., S. 28-33.

13 Mortimer, Rex: Class, Social Cleavage \& Indonesian Communism, S. 17 und Hawkins, Everett D.: Indonesia, in: Galenson, W. (ed.): Labor in Developing Economies, Berkeley, Los Angeles, 1962, S. 71-137, S. 95.

14 Rocamora, J.: Nationalism in Search of Ideology: The Indonesian Nationalist Party, 1946-1965, Ann Arbor, 1974, S. 398. 
von oben, organisiert und gelenkt von Parteikadern, deutlich. Enormen Zuwachs erhielt KBKI nach Mitte 1957, als die an Masjumi und PSI angelehnten Gewerkschaftsbünde wegen deren Beteiligung an Separationsbewegungen an Einfluß verloren. Es hatte die führende Rolle bei der Úbernahme der holländischen Firmen ab Dezember 1957 inne. ${ }^{15}$ Seine Ende der 50er Jahre relativ starke Position büßte KBKI aber ein, als zu Beginn der 60er Jahre die Zerrissenheit der PNI in der Frage der Zusammenarbeit mit den Kommunisten zu Spaltungen führten, so daß 1965 zwei nationalistische Gewerkschaftsbünde bestanden: KBKI und KBM (Kesatuan Buruh Marhaen - Gewerkschaft der Marhaenistischen Arbeiter) ${ }^{16}$ Beider Arbeit wurde seither aber wesentlich behindert einmal durch Säuberung von linksgerichteten Kräften, einmal dadurch daß Regierungsangestellten, von denen vorher ein bedeutender Teil Mitglieder von KBKI oder KBM waren, nur noch die Mitgliedschaft in der von der Regierung eingesetzten Beamten-Organisation gestattet war. $^{17}$

\subsection{Islamische Gewerkschaftsbünde}

Seit den späten 20er Jahren bis in die Zeit des Unabhängigkeitskampfes hatten die islamischen Führer der Arbeiterbewegung wenig Aufmerksamkeit geschenkt. Als sich die Führer der Masjumi-Partei (Madjelis Sjuro Muslimin Indonesia, Rat der Muslimischen Vereinigungen Indonesiens) 1947 trafen, hatte die kommunistisch beeinflußte Arbeiterbewegung schon einen enormen Aufschwung erlebt. Daher beschlossen sie, eine Gewerkschaft auf der Basis islamischer Prinzipien, z. B. Brüderlichkeit auch im Sinne einer Zusammenarbeit zwischen Arbeit und Kapital statt Klassenkampf, ${ }^{18}$ zu gründen, und es entstand im November 1948 SBII, Sarekat Buruh Islam Indonesia, Gewerkschaft Islamischer Arbeiter Indonesiens, mit einer unitarischen Struktur, d. h. der Beitritt erfolgt nur aufgrund des Bekenntnisses zum Islam. In der ständigen Konkurrenz zu den kommunistischen und nationalistischen Gewerkschaften entwickelte sich SBII bis Ende der 50er Jahre ungefähr zum zweitstärksten ${ }^{19}$ Gewerkschaftsbund. Als die Beteiligung von Masjumi an den regionalen Separationsbewegungen in Sulawesi (Celebes) und Sumatra sich negativ auf SBII auszuwirken begann, distanzierten sich die Führer von SBII von Masjumi und nahmen eine völlige Umstrukturierung (in ein föderatives System auf Industriebasis) vor, auch der Name wurde geändert: Er hieß jetzt GASBIINDO, Gabungan Serikat2 Buruh Islam Indonesia, Vereinigung der Islamischen Gewerkschaften Indonesiens. ${ }^{20}$

15 Ebda., S. 405.

16 Marhaenismus war eine Ideologie, die Sukarno entworfen hatte.

17 Capizzi, Elaine: De Vakbonden onder de Nieuwe Orde, S. 94-116, in: Wertheim, Ernst; Utrecht, Ernst; Pluvier, J. M. u. a.: Tien Jaar Onrecht in Indonesië, Amsterdam, 1976, S. 106 f.

18 Gemäß Programm von SBII, bei Sandra, Sejarah Pergerakan Buruh Indonesia, Jakarta. 1961 (Geschichte der Indonesischen Arbeiterbewegung), Anhang 8, S. 192 f.

19 Lt. Hawkins/McVey, op. cit. S. 260.

20 GASBIINDO (Hrsg.): Buku Peringatan Ulang Tahun keXV, Jakarta 1963 (Gedenkschrift zum 15. Jahrestag von GASBIINDO), S. 8. 
GASBIINDO verfolgte eine streng antikommunistische Linie und suchte dabei schon vor 1965 Rückendeckung beim Militär. ${ }^{21}$ 1965/66 beteiligte sich GASBIINDO massiv an der Bekämpfung und Ausschaltung von PKI und SOBSI ${ }^{22}$ und unterstützte im großen und ganzen Suharto und die "Neue Ordnung". Durch die Ausschaltung von SOBSI wurde GASBIINDO zum größten Gewerkschaftsbund, sein Führer Agus Sudono, der 1957 ein halbes Jahr in den USA in einem laborship training geschult worden war, baute diese Position systematisch aus und setzte sich mit Hilfe der Regierung, ${ }^{23}$ nachdem er gewerkschaftsinterne Opposition ausgeschaltet hatte, an die Spitze des neu geschaffenen Gewerkschaftsbundes, der zwar noch die religiösen Grundsätze des Glaubens an Einen Allmächtigen Gott und der Brüderlichkeit im Programm hatte, ansonsten aber der säkularen, technokratischen Regierungslinie entsprach.

Es bestanden noch einige andere, kleinere islamische Gewerkschaftsbünde, auf die hier nicht näher eingegangen zu werden braucht, da sie keine größere Rolle in der indonesischen Gewerkschaftsbewegung spielten.

\subsection{Gewerkschaftsbünde des Militärs}

Das Eingreifen des Militärs in die Gewerkschaftsbewegung, indem sie eigene Organisationen gründeten, hat v. a. zwei Voraussetzungen, einmal das mit "Dwi fungsi" (Doppelfunktion) umschriebene Selbstverständnis des Militärs, ${ }^{24}$ das auch in Sukarnos bereits erwähntem Konzept der Funktionalen Gruppen bestätigt wurde ${ }^{25}$ und das eine Beteiligung des Militärs an den gesellschaftlichen Prozessen des Landes, für dessen Freiheit es einst Seite an Seite mit der übrigen Bevölkerung gekämpft hatte, vorsah; zum anderen das Eindringen von Offizieren in die führenden Stellen, die v. a. in den verstaatlichten Plantagen nach der Nationalisierung der holländischen Unternehmen 1957 frei geworden waren. ${ }^{26} 1961$ entstand in diesem Zusammenhang die Plantagengewerkschaft PERKAPPEN, Persatuan Karyawan Perusahan Perkebunan Negara - Vereinigung der Arbeitnehmer der Staatlichen Plantagenunternehmen, die nur der Form und dem Namen nach eine Gewerkschaft war. Tatsächlich waren die Vorsitzenden der örtlichen PER-

21 Gemäß einem unveröffentlichten Manuskript über die indonesischen Gewerkschaften.

22 Castles, Stephen: GOLKAR und die Konsolidierung der Macht, in: Turlach, M. (Hrsg.) Gesellschaft und Politik in Süd- und Südostasien. Bonn-Bad Godesberg, 1972 (Schriftenreihe des Forschungsinstituts der Friedrich-Ebert-Stiftung, Bd. 93) S. 269-282, S. 275 ff. und Friedrich-Ebert-Stiftung (Hrsg.). Die Rolle gesellschaftlicher Gruppen im Entwicklungsprozeß Indonesiens, den Philippinen und Singapur, Bonn-Bad Godesberg, Aug. 1972, D. 219 ff.

23 So begrüßte Agus Sudono im Oktober 1969 vor der Gründung von MPBI (s. S. ?? f.) diese von der Regierung eingesetzte Organisation. Kompaß, 10. 10. 69 (Indonesische Tageszeitung).

24 S. Sielaff, Rüdiger: Die gesellschaftspolitischen Vorstellungen des Militärs und die Veränderung der politischen Strukturen in Indonesien, S. 283-298), in: Turlach, op. cit., S. 283 ff.

25 Sukarno: The Armed Forces as an Instrument of Revolution, 1961, in: Feith, H. and Castles, L. (ed.): Indonesian Political Thinking 1945-1965, Ithaca 1970, S. 434-435.

26 FES, op. cit., S. $211 \mathrm{ff}$. 
KAPPEN-Organisationen oft die Manager der entsprechenden Plantagen, ${ }^{27}$ und die hohen Mitgliedszahlen, von denen die Rede war, dürften unter erheblichem Druck entstanden sein. ${ }^{28}$ Als nach 1965/66 die kommunistisch orientierten Plantagengewerkschaften ausgeschaltet waren, behauptete PERKAPPEN, $50 \%$ aller Plantagenarbeiter Nord-Sumatras zu organisieren. PERKAPPEN war die wichtigste Einzelgewerkschaft der 1961 unter der Schirmherrschaft von Gen. Nasution und Gen. Yani als antikommunistische Arbeiterorganisation gegründeten SOKSI - Sentral Organisasi Karyawan Sosialis Indonesia, Zentrale der Sozialistischen Arbeitnehmerorganisationen Indonesiens.

Wenn sich auch.SOKSI mit Militär und Regierung identifiziert hatte, ${ }^{29}$ so wurde sie doch nach 65/66 durch den Vereinheitlichungsprozeß genau so absorbiert wie die anderen noch existierenden Gewerkschaften auch.

Da sie außer im Plantagenbereich mit PERKAPPEN kaum echte gewerkschaftliche Arbeit geleistet hatte und auch weiter keine Basis besaß, ihr zudem die Regierungsangestellten, die seit 1971 Mitglieder der regierungseigenen Gruppen werden mußten, entzogen waren, war SOKSI nicht geeignet, wie man vielleicht hätte vermuten können, zum einheitlichen Dachverband der "orde baru" zu werden.

\subsection{Unabhängige Gewerkschaften}

Auch nach dem Unabhängigkeitskampf gab es unter den indonesischen Gewerkschaftern eine Gruppe, die sich für von parteipolitischem Einfluß freie Gewerkschaften einsetzte. Der dauerhafteste der unabhängigen Gewerkschaftsbünde war GSBI, Gabungen Serikat Buruh Indonesia, Verband der Gewerkschaften Indonesiens, der 1949 aus dem Zusammenschluß zweier kleinerer Gewerkschaftsbünde entstanden war und eigentlich eine Vorstufe zu einem Dachverband sein sollte. ${ }^{30}$ Uber alle nur schwer überschaubaren Bündnisse, Spaltungen und Zusammenschlüsse hinweg hielt sich GSBI als kleiner, antikommunistisch eingestellter Gewerkschaftsbund, der sich zu den Prinzipien eines demokratischen Sozialismus bekannte. ${ }^{31}$ Zeitweise gehörte die große und mächtige Lehrergewerkschaft PGRI, Persatuan Guru Republik Indonesia, zu ihm, die zusammen mit der Eisenbahnergewerkschaft PBKA, Persatuan Buruh Kereta Api, zu den wenigen unabhängigen Einzelgewerkschaften Indonesiens gehörte, die echte Basisarbeit geleistet haben. ${ }^{32}$

27 Hasibuan, Sayuti: Political Unionism and Economic Development in Indonesia: Case Study North Sumatra, Michigan, 1976, S. 42.

28 FES, op. cit., $260 \mathrm{f}$.

29 Hasibuan, op. cit., S. 151. (Gemeint ist hier die Regierung der "Neuen Ordnung".)

30 Sandra, op. cit., S. 81 f.

31 Tedjasukmana, op. cit., S. 38 u. 57.

32 PBKA war eine der wenigen Gewerkschaften Indonesiens, die regelmäßig Beiträge ihrer Mitglieder einzog, damit unabhängig und in der Lage war, wirklich etwas für ihre Mitglieder zu tun. (Hawkins/Galenson, op. cit., S. 99) PBKA hatte eine Unfallversicherung und eine Allgemeine Krankenversicherung, eine Arbeiterschule in Bandung, einen Badan Sosial Pusat (Zentrale Körperschaft für Soziales), der kleine Ersparnisse der 
PGRI wurde nach 1965/66, weil es sich einer Eingliederung in die von der Regierung eingesetzte Einheitsgewerkschaft widersetzte ${ }^{33} 1974$ zu einem Fachverband gemacht. PBKA, das sehr unter der Konkurrenz der an SOBSI angeschlossenen Eisenbahnergewerkschaft SBKA, Serikat Buruh Kereta Api, gelitten hatte und vor 1965 fast zum Erliegen gekommen war, ${ }^{34}$ erholte sich nach 1966 wieder, bis es 1973 in der Industriegewerkschaft von FBSI aufging.

\subsection{Arbeitnehmer-Organisationen der Regierung}

Am 6. 11.57 wurde auf Anordnung von Stabschef Nasution eine Kommission für die Zusammenarbeit zwischen Militär und verschiedenen Verbänden, darunter auch Gewerkschaften, gegründet. Aus dieser Kommission ging 1964 das Gemeinsame Sekretariat der Funktionellen Gruppen, genannt SEKBER GOLKAR, hervor. Auch hier fanden Mitglieder der verbotenen Parteien PSI und MASJUMI "eine politische Heimat " ${ }^{35}$ im wesentlichen aber war SEKBER GOLKAR nicht parteipolitisch orientiert. Als Zusammenschluß von über 200 Gruppen aus den verschiedensten Bereichen der Gesellschaft war es auch kein monolithischer Block ${ }^{36}$ und entwickelte sich erst nach 1965/66 zu der Regierungsorganisation, die es heute ist. Vor 1965/66 gehörte als gewerkschaftliche Organisation SOKSI dazu, danach schlossen sich noch zahlreiche andere Gewerkschaften aus Angst vor Verfolgung durch die Militärregierung an, u. a. GASBIINDO, SOBRI, KBKI, PGRI.

Nach seiner Reorganisation im Sinne der radikalen Kräfte der "Neuen Ordnung" 1969 nahm es durch Straffung der Organisationsform, wodurch GOLKAR jetzt statt einer Federation ein zentralgeleiteter Verband ist, mehr den Charakter einer Partei an. ${ }^{37}$

Von ihm ging der Versuch aus, die bestehenden Gewerkschaften in einem unpolitischen Bund zusammenzuschließen. Seine Organisation für die Regierungsangestellten wurde durch Präsidenten-Erlaß vom 29. 11. 71 für diese zur Pflichtorganisation, d. h. alle bei der Regierung Beschäftigten mußten bei KORPRI, Korps Pegawai Republik Indonesia - Verband der Angestellten der Indonesischen Republik - organisiert sein, die Mitgliedschaft bei einer Gewerkschaft war ihnen verboten. Eine echte Vertretung ihrer Interessen finden sie in dieser Organisation nicht, sie haben unter dem Schlagwort der "Monoloyalität“ ihre Interessen dem Staatsinteresse unterzuordnen. ${ }^{38}$

Arbeiter sammelte und in genossenschaftlichen Unternehmungen, wie Reismühle, Batik-Betrieb u. a., investierte. PBKA (Hrsg.) Laporan Kerdja 3 Tahun 1955-1958 untuk Kongres keV, Bandung 1959 (Tätigkeitsbericht).

33 Persönliche Mitteilung von Radjamas, einem der Vorsitzenden von FBSI.

34 Unveröffentlichtes Manuskript über die indonesischen Gewerkschaften.

35 Sielaff/Turlach, op. cit., S. 290.

36 V. d. Kroef, Justus: Indonesia since Sukarno, Singapore, 1971, S. 22.

37 In dieser Form nahm GOLKAR an der Wahl am 3. 7. 71 teil und errang 236 von 360 Mandaten. S. Castles/ Turlach, op. cit., S. 269 u. 280.

38 So ist ihnen auch die Zugehörigkeit zu einer Partei verboten. Kompaß, 31. 12. 1974. 


\section{Einigungsbestrebungen}

Bei dieser Vielfalt in der Gewerkschaftsbewegung - und Vielfalt ist in Indonesien eine Konstante der gesellschaftlichen Entwicklung und im Staatsmotto "Einheit in der Vielfalt" zum Ausdruck gebracht - ist es nicht verwunderlich, daß es seit den ersten Gewerkschaftsgründungen Versuche gegeben hat, einen alle bestehenden Gewerkschaften und Gewerkschaftsbünde umfassenden Dachverband zu schaffen. Zunächst gingen die Versuche dazu (1919, ${ }^{39} 1922$ z. B.) von den Gewerkschaften selbst aus, ohne daß je die Zersplitterung länger als für im günstigsten Fall vier Jahre überwunden werden konnte. 1959, mit der Proklamierung der Gelenkten Demokratie, begann auch die Regierung Initiativen in dieser Richtung zu entfalten. Im Rahmen von Sukarnos Vorstellungen einer Gliederung der Gesellschaft in "Funktionelle Gruppen" (Golongan Fungsionil) wurde KOBI, Koordinasi Buruh Indonesia ${ }^{40}$ geschaffen, ein Koordinierungsausschuß, der nicht nur der Regierung als ein Verhandlungspartner dienen, sondern auch ein Gegengewicht zu dem aufgrund der Initiative des Industrieministers einige Monte zuvor entstandenen Unternehmerverband (Gabungan 2 Perusahaan Sedjenis) bilden sollte. Dieser Ausschuß führte aber noch nicht zu dem schon so oft angestrebten Ziel einer Bündelung der divergierenden Gewerkschaftsorganisationen. Auch die auf die direkte Einflußnahme des damaligen Arbeitsministers Ahem Erningpradja (PNI) zurückgehende Gründung von OPPI, ${ }^{41}$ Organisasi Persatuan Pekerdja Indonesia, - Organisation Vereinigter Arbeiter Indonesiens -, war nicht von Dauer, ebenso wie sein erneuter Anlauf - SBGSI, Sekretariat Bersama Gabungan Serikat Indonesia, ein gemeinsames Sekretariat aller damals bestehenden großen Gewerkschaftsbünde, das allerdings nicht nur im Interesse einer einheitlichen Gewerkschaftsbewegung, ${ }^{42}$ sondern v. a. zu propagandistischen Zwecken gegründet worden war. ${ }^{43}$

SBGSI hatte während des West-Irian-Konflikts bereits eng mit dem Militär zusammengearbeitet. Nach dessen Beilegung ergriffen Offiziere die Gelegenheit, in der Gewerkschaftsbewegung Fuß zu fassen: Sie riefen neue gewerkschaftliche Organisationen ins Leben, v. a. im Plantagenbereich, in dem viele Offiziere die durch die Nationalisierungskampagne 1957, in der die holländischen Besitzungen übernommen worden waren, frei gewordenen Stellen eingenommen hatten. An diese Entwicklung konnte die Militärregierung nach 1965 anknüpfen. Nach einem kurzen Wiederaufleben von SBGSI ${ }^{44}$ wurde 1968 unter dem Vorsitz von Gen. Major Sukowati eine Kommission (BAKER PROBI Badan Kerdja Projek Buruh) ins Leben gerufen, die v. a. auf Initiative des IBFG zustandegekommen war und als Anlauf- und Koordinierungsstelle für Hilfe aus dem westli-

39 In dem ersten Dachverband war es der islamisch-kommunistische Gegensatz, der zur Spaltung führte.

40 Sandra, op. cit., S. $141 \mathrm{f}$.

41 Ebda., S. $148 \mathrm{f}$.

42 US Department of Labor (ed.): Labor in Indonesia, Washington, 1963, S. 50.

43 Unveröffentlichtes Manuskript.

44 FES, op. cit., S. 271. 
chen ${ }^{45}$ Ausland an die indonesischen Gewerkschaften fungieren sollte. Daß Generalmajor Sukowati Vorsitzender von BAKER PROBI wurde, zeigt deutlich die beherrschende Position der Regierung und von GOLKAR, der regierungseigenen Massenorganisation, deren Vorsitzender Sukowati ist. Aus diesem Grunde und wegen des ausländischen Einflusses lehnten viele Gewerkschafter BAKER PROBI ab. ${ }^{46}$ So war auch diese Kommission nicht mehr als ein kurzlebiger Anlauf.

Am 1. 11. 1969 unternahm das Arbeitsministerium einen erneuten Versuch, die durch politische und persönliche Differenzen gespaltene Gewerkschaftsbewegung in einem Dachverband zu einen: 25 Gewerkschaften wurden im Verhandlungsrat für die Arbeiter Indonesiens - MPBI, Madjelis Permusjawaratan Buruh Indonesia, zusammengeschlossen. Daß dieser Rat immer mehr zu einem Instrument des Arbeitsministeriums wurde, wird schon daraus deutlich, daß die Büros der örtlichen Vertretungen in den Büros der Verwaltungsstellen des Arbeitsministeriums untergebracht waren. ${ }^{47}$ Aber das Mißtrauen der Gewerkschaften, von der Regierung vereinnahmt zu werden, war nicht der einzige Grund dafür, daß auch MPBI noch nicht zu einem dauerhaften Dachverband wurde: Die Gewerkschaftsführer konnten sich über grundlegende Verfahrensfragen, wie die Bindungskraft der Beschlüsse von MPBI für die Mitgliedsgewerkschaften und die Vertretungsmodi für die großen und kleinen Gewerkschaften nicht einigen..$^{48}$ So war MPBI nie mehr als ein Treffpunkt und Diskussionsforum. ${ }^{49}$

\section{Der bestehende Dachverband der indonesischen Gewerkschaften/FBSI}

War bei den vier zuletzt besprochenen Einigungsversuchen der indonesischen Gewerkschaften die Regierung offen als Initiator aufgetreten, so war es nun der islamische Gewerkschaftsbund GASBIINDO, v. a. durch seinen Vorsitzenden Agus Sudono, der die Schritte zu der Schaffung des heute noch bestehenden Dachverbandes unternahm. Zumindest sollte es wohl so aussehen, daß die Initiative von einer der größeren, auch an MPBI und den vergleichbaren vorausgegangenen Organisationen beteiligten Gewerkschaften kam, um einerseits dem von den Gewerkschaften bisher an den Tag gelegten Mißtrauen den Boden zu entziehen und andererseits der kritischen Offentlichkeit und dem Ausland gegenüber auf einen eigenständigen Gewerkschaftsverband weisen zu können. Daß es gerade der islamische Gewerkschaftsbund GASBIINDO war, der sich zum Vorreiter dieses erneuten Versuches machte, ist Ausdruck einer Entwicklung, die schon lange vor 1965/66 eingesetzt hatte: Erstens hatte GASBIINDO einen streng antikommunistischen Kurs verfochten, ${ }^{50}$ zweitens hatte es sich zu diesem Zweck bereits mit dem Mi-

45 V. a. aus der BRD und den USA.

46 FES, op. cit., S. 266.

47 FES, op. cit., S. 267.

48 Unveröffentlichtes Manuskript.

49 Sukarno: This is the FBSI, Jakarta 1974, S. 8.

50 FES, op. cit., S. 219 ff. 
litär verbündet ${ }^{51}$ und war 1965/66 dementsprechend aktiv an der Bekämpfung der Kommunisten beteiligt.

Auch hinter dieser Initiative stand aber die indonesische Militärregierung. Anläßlich eines Besuches der nationalistischen Gewerkschaftsvertreter von KBM bei Arbeitsminister Sadli im Nobember 1971 betonte er, daß die Regierung keinerlei drastische Maßnahmen gegen die Gewerkschaften ergreifen wolle, daß es allerdings richtig sei, daß es Pläne zu einer Vereinfachung gäbe. ${ }^{52}$ Ein weiteres Indiz ist der parallel verlaufende Einigungsprozeß dem die nach 1965/66 verbliebenen Parteien unterworfen wurden und an dessen Ende 1973 der Zusammenschluß von PNI, PARKINDO, Partai Katolik, IPKI und Partai Murba zu Partai Demokrasi Indonesia - PDI - und der von Nahdutal Ulama, Partai Muslimin Indonesia, Partai Sarekat Islam und PERTI zu Partai Persatuan Pembangunan - PPP - (Entwicklungspartei) stand. ${ }^{53}$

Als Forum für den neuen Einigungsanlauf im Gewerkschaftsbereich wurde der formell noch bis zum 26. 3. 1973 bestehende Verhandlungsrat MPBI benutzt. Auf einem Seminar, das von MPBI und der Stiftung Indonesische Arbeitskräfte der Friedrich EbertStiftung (Yayasan Tenaga Kerja Indonesia - YTKI) vom 21. bis 28. 10. 1971 in Jakarta abgehalten wurde, trug Agus Sudono folgende Analyse der indonesischen Gewerkschaften vor:

1. Sie seien politisch noch nicht unabhängig (gemeint ist damit, frei von parteipolitischer Bindung) und

2. finanziell noch nicht unabhängig.

3. Es gäbe noch interne und externe Faktoren, die einer Einigung im Wege stünden. ${ }^{54}$ Um den indonesischen Gewerkschaften den Anschluß an die allgemeine Entwicklung zu ermöglichen, die laut Agus Sudono in Richtung einer modernen, demokratischen Industriegesellschaft auf der Grundlage von Pancasila und der Verfassung von 1945 geht, müßten folgende Veränderungen vollzogen werden:

1. Die Arbeiterbewegung muß völlig frei von jeglicher politischer Macht, d. h. sie muß eine reine Arbeiterbewegung sein.

2. Sie darf von keiner außerhalb stehenden Geldquelle abhängig sein, muß also die Beitragserhebung intensivieren, und zwar durch ein check-off-System.

3. Die Schwerpunkte ihrer Aktivität müssen im sozial-ökonomischen Bereich liegen.

4. Die bestehenden Gewerkschaften müssen neu geordnet und dadurch daß sie sich einander annähern oder durch Gesetze als einer "social-engeneering" Maßnahme, nämlich indem ein umfassendes Gewerkschaftsgesetz herausgebracht wird, geeint werden.

5. Die Struktur der indonesischen Arbeiterbewegung muß reorganisiert werden, und zwar soll es für jede Branche nur eine Gewerkschaft geben (Industriegewerkschaft).

51 Unveröffentlichtes Manuskript.

52 Кompaß, 16. 11.71.

53 Vgl. auch v.d. Kroef, op. cit., S. 46 f.

54 Sudono/Sej., op. cit., S. 2.

55 Ebda., S. 3 f. 
Die Industriegewerkschaften sollen dann in einem Kongreß der Indonesischen Gewerkschaften/Bund der Indonesischen Gewerkschaften zusammengefaßt werden.

6. Dieser Gewerkschaftskongreß sollte zuallererst die Befähigung der Arbeiterführer fördern, damit sie den Forderungen ihrer Zeit begegnen können. Die Gewerkschaften müssen das Verantwortungsgefühl ihrer Mitglieder fördern gegenüber
a) ihrem Arbeitsplatz
b) ihrer Familie
c) ihrer Organisation
d) ihrer Gesellschaft, dem Land und Volk
e) dem Einen und Allmächtigen Gott.

Das Organisationsschema für die geplante Neuordnung der Gewerkschaften hat Agus Sudono von GASBIINDO übernommen, das ja schon $1960 \mathrm{zu}$ einem Bund von Industriegewerkschaften geworden war. Aber auch das Beispiel des DGB war ein bedeutender Faktor für die Wahl dieser Organisationsform. Anregungen dazu dürften wohl bereits innerhalb von BAKER PROBI (1968) gegeben worden sein, ${ }^{56}$ später (1971) wurde die heute noch bestehende, bereits erwähnte Stiftung YTKI gegründet, die tripartite strukturiert ist, d. h. in ihr wirken Vertreter von Arbeitgebern, Arbeitnehmern und Regierung bei der Klärung von Beschäftigungsproblemen zusammen.

Mit der Forderung der Loyalität gegenüber dem Einen und Allmächtigen Gott ist dem Anliegen der islamischen Kräfte Genüge getan, ansonsten hat der in den darauf folgenden Monaten auf der Grundlage dieser Vorstellungen geschaffene Gewerkschaftsbund aber durchaus säkularen Charakter.

Die Forderung, daß die Arbeiterbewegung frei von politischem Einfluß sein müsse, hatte Arbeitsminister Sadli in dem schon erwähnten Gespräch mit KBM ebenfalls erhoben und bekräftigte sie noch einmal im Februar des folgenden Jahres, ebenso wie die nach ihrer rein sozial-ökonomischen Ausrichtung. ${ }^{57}$

In der MPBI-Plenarsitzung vom 24. bis 26. 5. 1972, an der alle damals nach bestehenden wichtigeren Gewerkschaften und Gewerkschaftsbünde teilnahmen ${ }^{58}$ wurde eine Charta zur "Erneuerung und Vereinheitlichung der bestehenden Gewerkschaften Indonesiens" verabschiedet. Getreu der von der Regierung Suharto ausgegebenen Parole "Entwicklung statt Politik" sollten die Gewerkschaften eine rein sozio-ökonomische Funktion haben und an Entwicklung orientiert sein. ${ }^{59}$

Noch ein knappes Jahr verging, bis am 20. 2. 1973 mit der "Deklaration zur Einheit der Arbeiter Ganz Indonesiens ${ }^{\prime 60}$ der neue Gewerkschaftsbund FBSI, Federasi Buruh Seluruh Indonesia, Bund der Arbeiter Ganz Indonesiens, gegründet wurde. Am 8. 3. 1973 er-

56 In einem Artikel in der Tageszeitung "Kompaß" weist Arbeitsminister Sadli ebenfalls darauf hin, daß FBSI nach dem Muster des DGB strukturiert ist. Kompaß, 7. 3. 1973.

57 Kompaß, 6. 2. 1972.

58 Dieser Sitzung ging ein Seminar vom Arbeitsministerium in Zusammenarbeit mit YTKI/FES voraus.

59 Charta, wiedergegeben bei Sudono, Sej., op. cit., Anhang 2.

60 "Deklarasi Persatuan Buruh Seluruh Indonesia" vom 20. 2. 1973 bei A. Sudono, Sej., op. cit., Anhang 3. 
klärte der Vorstand von MPBI sein Einverständnis mit dieser Gründung, einstimmig, und löste sich am 20.3. 73 auf, um gleichzeitig in FBSI aufzugehen. ${ }^{61}$

Erster Vorsitzender von FBSI wurde Agus Sudono, weitere Vorsitzende Sukijat, Rasjid St. Radjamas, Drs. Sutanto Martoprasono und Adolf Rachman, die alle schon im vorbereitenden Komitee von MPBI tätig waren. Agus Sudono und Sutanto kommen von islamischen Gewerkschaftsbünden, Rachman und Sukijat von regierungsnahen Organisationen und Radjamas vom nationalistischen Gewerkschaftsbund KBM. Der Generalsekretär, Drs. Sukarno, gehörte dem militärischen Nachrichtendienst OPSUS ${ }^{62}$ an. Auffallend und bezeichnend für den Charakter des neuen Gewerkschaftsbundes ist auch, wie viele sogenannte "Technokraten", junge Akademiker, die meist in Amerika ${ }^{63}$ studiert hatten, in seiner Organisation oder der einer der Industriegewerkschaften, Posten erhalten haben. Wenn auch die technokratische Vorgehensweise nicht kritisch genug gesehen werden kann, v. a. im Hinblick auf eine freie Entfaltung von Basisaktivitäten, so kann man doch den Technokraten in Indonesien, zumindest in den ersten Jahren der "Neuen Ordnung" nach 1965/66 nicht jegliche gute Absicht absprechen. Viele Intellektuelle, unter ihnen viele Wirtschaftswissenschaftler, hatten den Kurs Sukarnos, der die wirtschaftliche Entwicklung des Landes vernachlässigt hatte, mit großer Skepsis betrachtet und heftig kritisiert. ${ }^{64}$ Unter der "Neuen Ordnung" nun glaubten sie, endlich etwas für die wirtschaftliche Entwicklung des Landes leisten zu können, und es besteht auch kein Zweifel, daß viele Bemühungen in dieser Richtung unternommen wurden. Ebenso besteht aber auch kein Zweifel, daß diese Bemühungen zwar zu einem Anstieg der Wachstumsrate des Bruttoinlandsproduktes von 1,96\% zwischen 1960 und 1965 auf 7,43\% zwischen 1969 und 1973 führten, aber daß die Massen des indonesischen Volkes davon nicht profitierten.

Genau dieselbe Diskrepanz besteht zwischen den Programmen und Organisationsplänen von FBSI und der Realität, der die indonesischen Arbeiter und Angestellten täglich begegnen müssen.

Die "ideelle" Grundlage für die von FBSI aufgestellten Prinzipien ist die bereits erwähnte Pancasila-Ideologie, ${ }^{65}$ die, von Sukarno 1945 propagiert, in der Vor-Gestapu-Zeit in der Gewerkschaftsbewegung keine besondere Rolle gespielt hatte:

Die Pancasila - Fünf Grundpfeiler - sind:

1. Nationalismus, 2. Internationalismus, 3. Permusyawaratan (Konsensus), 4. Soziale Gerechtigkeit, 5. Glaube an Einen Allmächtigen Gott.

61 "Penyataan Dukungan" und "Penyataan MPBI", Sudono/Sej., op. cit., Anh. 4 u.

62 Cappizzi, op. cit., S. 109.

63 Da v. a. an der University of Berkeley. Man spricht in Indonesien von einer "Berkeley-Mafia". Uber die Rolle, die die amerikanischen Universitäten bei der Vorbereitung der Etablierung der "Neuen Ordnung" gespielt haben, s. Ransom, David: The Berkeley Mafia and the Indonesian Massacre. S. 28-29 u. 40-49, in: Ramparts: The Lesson of a Massacre, Okt. 1970.

64 z. B. Mohammad Sadli: Inflation, the Drifting Kite, in: Feith, op. cit., 4. Moh. Sadli wurde unter der "Neuen Ordnung" Arbeitsminister.

65 Sukarno: The Pantja Sila, 1945, in: Feith. 
Analog dazu, der in Indonesien so beliebten Zahlenmagie treu bleibend, entwickelte FBSI "Hubungan Perburuhan Pancasila", Pancasila-Arbeitsbeziehungen:

1. In jedem Betrieb sollen "Bipartite"-Kommissionen eingerichtet werden, in denen Arbeiter und Unternehmer vertreten sind.

2. Das Tarifvertragswesen soll ausgebaut werden.

3. Für die Bildung der Arbeiter sollen Möglichkeiten geschaffen werden.

4. Es soll ein "Badan Peradilan Perburuhan" eingerichtet werden, eine Kommission für Arbeitsgerechtigkeit.

5. Die Arbeitsgesetzgebung soll ausgebaut werden.

Mit der Propagierung dieser Grundsätze gibt man sich viel Mühe: Allein, um die Frage zu ergründen, was diese konkret für die gewerkschaftliche Arbeit bedeuten sollen, wurden verschiedene Seminare veranstaltet, z. B. am 28. 11. 73 und vom 4. bis 7. 12. 1974 in Jakarta. Diese Grundsätze sollten zur Grundlage einer umfassenden Arbeitsgesetzgebung werden, was bis Oktober 1978 allerdings noch nicht verwirklicht war.

Für die tatsächliche Existenz der Bipartite-Kommissionen gibt es bisher keine Anzeichen. Im Bereich der Tarifverträge ist am ehesten etwas erreicht worden: Bis zum 20. 2. $1978^{66}$ waren 194 Tarifverträge abgeschlossen, die ungefähr 1340 Unternehmen (von 110.000 in Frage kommenden) abdeckten. Auch die "Kommission für Arbeitsgerechtigkeit" ist inzwischen ins Leben gerufen - sie dokumentiert mehr die hoffnungslose Lage der Arbeiter, als die konkreten Möglichkeiten zu ihrer Veränderung, da ja für Konflikte im Arbeitsbereich die seit 1951 bestehende Schlichtungskommission P4 ${ }^{67}$ zuständig ist. Verallgemeinernd kann man sagen, daß P4 in der Vor-Gestapu-Zeit eher zugunsten der Arbeiter entschieden hat, wogegen heute die Arbeiter allgemein über eine Benachteiligung in dieser Kommission klagen.

Was die Ausbildung anbelangt, so erklärte Agus Sudono, ${ }^{68}$ daß den noch immer spürbaren Auswirkungen der holländischen Kolonialzeit mit ihrer einseitigen Ausrichtung auf die white-collar-jobs entgegengewirkt werden müsse, es sollten künftig sowohl theoretische als auch praktische berufliche Kenntnisse vermittelt werden. Man arbeite auch in der Erwachsenenbildung mit der Friedrich-Ebert-Stiftung zusammen.

Diese Ausführungen leiten schon zu dem Programm ${ }^{69}$ von FBSI über, das auf die Fünf Grundsätze Fünf Aufgaben (Panca Karya) folgen läßt:

1. Entwicklung und Konsolidierung der Organisation.

2. Erhöhung der Partizipation der Arbeiter bei der Steigerung der Produktion im Rahmen der Entwicklung.

66 Lt. einer Rede von Agus Sudono anläßlich des V. Jahrestages v. FBSI.

67 Panitia Penjelesaian Perselisihan Perburuhan - Kommission für die Schlichtung von Arbeitskonflikten. Zusammengesetzt aus 5 Arbeitsvertretern, 5 Unternehmervertretern und 5 Regierungsvertretern. Hidajat, M. S.: Dasar2 Hubungan Perburuhan di Indonesia. Erlangga, 1970, S. 36 ff. (Grundlagen der Arbeitsbeziehungen in Indonesien).

68 In einem Gespräch mit der Verfasserin am 30. 8. 1975.

69 Programm Umum FBSI "Panca Karya" vom 11. 3. 1973, Sukarno/FBSI, op. cit., S. 27 f. 
3. Im Einklang mit den Grundsätzen der Gerechtigkeit die Rechte und Interessen der Arbeiter verteidigen.

4. In den Bemühungen zur Bewältigung der Arbeitslosigkeit und der Erweiterung der Arbeitsbeschaffung aktiv werden.

5. Die Zusammenarbeit mit internationalen Gewerkschaften in Einklang mit der Außenpolitik der Regierung, die unter dem Motto "Frei und Aktiv" steht, entfalten.

Und wie sieht es nun mit der Wahrnehmung dieser Aufgaben in der Realität aus? Natürlich wird niemand erwarten, daß die Führer dieses erst 1973 geschaffenen Gewerkschaftsbundes angesichts der enormen Probleme der indonesischen Arbeitswelt innerhalb weniger Jahre Wunder wirken können. Auch wenn man einmal davon absieht, daß schon die Prämisse

- ein Gewerkschaftsbund, von oben für ${ }^{70}$ die Arbeiter geschaffen, Führer, die sich bestimmte Aufgaben gesetzt haben - falsch ist, und diese Gewerkschaften an ihren eigenen Äußerungen mißt, müssen folgende Punkte kritisch hervorgehoben werden:

1. Die Entfaltung der Organisation geschieht keinesfalls auf demokratische Weise (lt. Artikel der FBSI-Statuten): ${ }^{71}$ Die Betriebsgruppen bilden sich nicht selbst von der Basis her, sondern FBSI-Kader gehen in die Betriebe und drängen die Betriebsleitung, in ihrem Betrieb eine Gruppe (entsprechend dem Industriezweig) einzurichten und die Beiträge in einem Einzugsverfahren zu erheben. ${ }^{72}$ Hinzu kommt, daß bis auf Kreisebene jede Organisation sich zwei Drittel ihrer Führungsleute vom Geheimdienst (BAKIN) bestätigen lassen muß.

Seit April 1973 entstanden 21 Industriegewerkschaften, denen die zu der Zeit bestehenden Einzelgewerkschaften eingegliedert wurden. ${ }^{73}$

2. Abgesehen davon, daß es nicht die Aufgabe einer Gewerkschaft ist, ihre Mitglieder zu höherer Produktivität aufzurufen, geht eine solche Forderung völlig an der Realität vorbei, die durch Faktoren gekennzeichnet ist, von denen hier nur die wichtigsten aufgeführt werden:

a) Die Löhne sind so niedrig, daß sie nicht einmal das Existenzminimum decken, die Mehrzahl der Arbeiter also völlig unzureichend ernährt ist, dadurch anfällig für

70 Wobei man nicht einmal das "für" unwidersprochen stehen lassen kann . .

71 Art. 6, Abs. 4 "To create sound and harmonious industrial relations by way of defending and fighting for the interest of the workers toward the realization of social, legal and democratic orderlines. Sukarno/FBSI, op. cit., S. $21 \mathrm{ff}$.

72 Ich habe gehört, daß ein Betriebsleiter, der einwendete, die Arbeiter hätten nicht genug Geld für Beiträge und auch keine Bereitschaft zu zahlen, als Antwort erhielt, dann solle der Betrieb sie bezahlen.

731975 hatten die zunächst 18 Industriegewerkschaften insgesamt 1385 Niederlassungen (gemäß Suara Buruh Aug. 1975, Zeitschrift von FBSI, S. 27). 1977 erfolgte die Trennung der Transportgewerkschaft in drei Industriegewerkschaften (Land, Luft, Wasser). Bis zum 10. 7. 1978 hatten die inzwischen 21 Industriegewerkschaften insgesamt 4489 Niederlassungen (gemäß bei FBSI eingegangenen Berichten). Mitgliedszahlen waren bei FBSI nicht in Erfahrung zu bringen. Ein Organisationsschema findet sich bei "Kumpulan Bahan2 Penting FBSI", Biro Administrasi \& Logistik DPP FBSI, Jakarta, Nov. 1975 (Sammlung von wichtigen Materialien von FBSI). 
Krankheiten (Tuberkulose ist z. B. in Indonesien immer noch erschreckend weit verbreitet), für welche auch nicht genügend vorgesorgt ist. ${ }^{74}$

b) Ebenfalls durch die geringen Löhne bedingt, suchen sich viele Arbeiter und Angestellte zweite, dritte Beschäftigungen, um zu einem einigermaßen ausreichenden Verdienst zu kommen. Dies bedingt auch Fehlzeiten.

3. Die bedenklichste Situation herrscht in Indonesien in Bezug auf Arbeitslosigkeit - zu dem Heer der Millionen zählenden stoßen jährlich noch mindestens eine Million neue Arbeitssuchende hinzu. ${ }^{75}$ Gemäß dem letzten Fünfjahresplan REPELITA III (Rentjana Pembangunan Lima Tahun, 1980/1 - 1985/6) erwartet die Regierung 6-7 Mio. neue Arbeitssuchende. Es gibt Programme der Regierung, um dieses Problem anzupacken, sie werden aber keinen nennenswerten Effekt erzielen, solange die Regierung zwar auf der einen Seite von arbeitskräfteintensiven Industrien spricht, auf der anderen Seite aber kapitalintensiven Industrien Tür und Tor öffnet.

FBSI sagt zu diesen Problemen fast nichts, und wird wohl dazu auch nicht viel sagen können, da jede tiefergehende Diskussion dieser Fragen die ideologischen Grundlagen, auf denen die Regierung und - als ihr Geschöpf, auch er ruht, in Frage stellen würde.

5. Die Zusammenarbeit mit internationalen Gewerkschaftsorganisationen, IBFG, ASEAN-Gewerkschaften, ILO, hat sich gut entwickelt, wobei ja ILO ebenfalls nach dem tripartite System einer Vertretung von Arbeitnehmern, Arbeitgebern und Regierungsseite arbeitet.

Abschließend soll noch kurz die Frage angesprochen werden, ob die indonesischen Gewerkschaften als soziale Bewegung verstanden werden können, bzw. ob in Zukunft in ihrem Rahmen eine solche entstehen könnte.

\section{Die Gewerkschaften Indonesiens: Soziale Bewegungen?}

Bis 1950, als Indonesien seine Unabhängigkeit von der Holländischen Kolonialmacht erkämpft hatte, war die Arbeiterbewegung im wesentlichen ein Teil der Unabhängigkeitsbewegung, und die war - das wurde besonders in der entscheidenden Phase des Unabhängigkeitskampfes zwischen 1945 und 1950 deutlich - eine alle Bereiche der Gesellschaft umfassende Bewegung auf einer breiten Basis.

Zwischen 1950 und 1965 trifft auf Indonesien eher das zu, was Sombart über die europäischen Revolutionen des 18. und 19. Jhs. sagte, nämlich die Bourgeoisie holte "das Pro-

74 Erst seit 1977 gibt es eine gesetzliche Grundlage für eine umfassende Sozialversicherung: ASTEK (Asuransi Sosial Tenaga Kerja).

75 Der Zensus von 1971 gibt 2,24 Mio. Arbeitslose (8,83\%) an. Sundrum, R. M.: Unemployment in Indonesia. Analysis of Cenus Data, in: Ekonomi dan Keuangan Indonesia, Vol. XXIII, No. 3, Sept. 1975, S. 261-267, S. $261 \mathrm{f}$.

76 Sombart, Werner, Sozialismus und soziale Bewegungen, Jena, 1908, S. 151, zitiert bei Wilkinson, Paul: Soziale Bewegungen, München, 1974, S. 156. 
letariat wie einen Teufel aus dem Kasten und ließ es verschwinden, sobald sie ihre Zwekke mit seiner Hilfe erreicht hatte. ${ }^{76}$ Obwohl die Kommunistische Partei und der Kommunistische Gewerkschaftsbund SOBSI durchaus eine Massenbasis hatten, so entsprachen sie als Kaderorganisationen doch nicht einer sozialen Bewegung im Sinne eines von den Bürgern selbst ausgehenden bewußten Versuchs zur Umgestaltung einer den Grundbedürfnissen nicht gerecht werdenden Gesellschaft in eine solche, die die Voraussetzungen dazu erfüllt.

$\mathrm{Da}$ in diesem Sinne von den Gewerkschaften der "Neuen Ordnung" nicht von einer sozialen Bewegung die Rede sein kann, versteht sich aus allem Dargelegten von selbst.

Bleibt die Frage, ob sich im Rahmen dieser Struktur eine solche entwickeln könnte, und sie müßte wohl im Rahmen dieser Struktur entstehen, wenn man davon ausgeht, daß in einem durchbürokratischen Land, wie es Indonesien ist, eine Organisation zur Durchsetzung der Interessen der Arbeiter notwendig ist: Denn der Aufbau konkurrierender Gewerkschaften von der Basis her wurde seit 1975 durch ein geändertes Gesetz über die Zulassung von Gewerkschaften ${ }^{77}$ unmöglich gemacht. Nach diesem Gesetz werden nur noch solche Gewerkschaftsbünde zugelassen, die in mindestens 20 Provinzen jeweils mindestens 15 Einzelgewerkschaften haben und auf der Grundlage von Pancasila ruhen. Es ist offensichtlich, daß dieses Gesetz FBSI auf den Leib geschrieben ist und auf lange Sicht hin jede alternative Gewerkschaftsbewegung unmöglich macht.

Es gibt Anzeichen dafür, daß junge Gewerkschafter versuchen, innerhalb der vorgegebenen Strukturen echte Basisarbeit zu leisten, um die Arbeiter dazu anzuregen, ihre Probleme selbst anzupacken. Man kann nur hoffen, daß dies Anzeichen eines zukunftsweisenden Trends sind. 
lar to that attributed to Bolivia by Che Guevara. Indeed, Colombia plays a much more important role in Moscow's interests than Bolivia did. At long term, under the strategical aspect, Columbia is a more important footboard for the Soviet Union that a dwarf nation in the frontyard ot the USA.

Violence threatens to become an end in itself. In its intensified form, it is a component of the social character: The attempt to react to problems or rather to solve these comes to a standstill as violence is used. Revolution is its outlet. Restricted to the national environment, guerilla movements are historically out of date or condemned to failure for two reasons:

1. Syndicates and basic movements are more important than armed movements whenever they bring forth movements advocating general strikes as purposeful political instruments and conclude alliances with most of the relevant sectors of the society.

2. Very often, the opposition leaders lack the plan of a society based on new economic structures at all levels - within the society and in the international context. Today, revolution is no longer a national question. It can only occur within the framework of conditions on the world market which are undergoing a fundamental change.

\section{Indonesian Trade Union Movement}

\section{By Eva-Maria Garang-Schaarschmidt}

The development of the Indonesian trade union movement is connected with the people's fight for independence from Dutch colonialism. This fight united the diverging forces, of which Islam and communism, both strongly amalgated with nationalism, have been the main ones, at least at times, whereas after independence having been reached, a variety of trade unions and union federations were formed. Before 1965

- the PKI dominated federation SOBSI with a membership of about $60 \%$ of all organized workers

- the Islam orientated federation GASBIINDO,

- the PNI dominated KBKI

have been the most important ones among hundreds of registered unions and union federations. But military had also already as early as 1961 tried to take part in the trade union movement. The cleavage between Islam and Communism could not be overcome even during Guided Democracy; while communism allied itself with nationalism, Islam began to strengthen its position in associating with military. Although SOBSI has not been involved in the Coup d'Etat of 1965, it has been prohibited, many of its members have been prosecuted, killed or jailed.

The technocratically oriented "new order" government under Suharto enforced trials already initiated under "old order" government of Sukarno to reduce the variety of exi- 
sting trade unions to one centralized federation with a structure along industrial lines. After several failures, GASBIINDO acted as initiator of the 1975 founded FBSI - Federasi Buruh Seluruh Indonesia (All Indonesian Trade Union Federation). In its structure it follows the model of the German Federation DGB, in its program the Pancasila (FivePrinciples) ideology once created by Sukarno; i. e. in respect to labour problems Five Tasks, Five Principles, including cooperation with government and entrepreneurs instead of fighting for better conditions of the organized workers.

Althouhg structure and programme of FBSI are declaredly democratic in practice this federation under leadership of Agus Sudono, former leader of GASBIINDO, is not an organization by the workersf or the workers but in fact a government lead institution lakking, consequently, any mass backing. 